\title{
CONFLICTO VISIBLE E INVISIBLE. UNA PROPUESTA DE ACERCAMIENTO A LOS CONTACTOS ENTRE CAZADORES Y PRODUCTORES DURANTE LA TRANSICIÓN AL NEOLÍTICO
}

\author{
Carmen Cortés Echevarría ${ }^{1}$
}

\section{Resumen:}

La aparición y expansión del modo de vida neolítico nos pone frente a una situación mundial bastante peculiar. Dos formas de vida radicalmente diferentes en el ámbito económico comparten y se reparten el territorio. Con apenas vestigios atribuibles a una posible violencia directa entre ambas facciones, podemos intuir diferentes encuentros y desencuentros, y ausencia de ellos, entre cazadores y productores en el registro arqueológico. Pretendemos plantear aquí conceptos como contacto, resistencia o frontera, de forma que nos ayuden a dar una nueva visión en las relaciones grupales e intragrupales que se produjeron en la transición entre el mesolítico y el neolítico. De este momento de cohabitación pretendemos visibilizar relaciones prolongadas y contactos puntuales, pero también formas de conflicto muchas veces indirectos. Sea como fuere, el final de este período de coexistencia es claro: la práctica desaparición de las formas de vida puramente cazadoras-recolectoras y la paulatina adopción, a gran escala, antes o después, de lo que entendemos como formas de vida neolíticas.

\section{Palabras Clave:}

Neolítico/Mesolítico, Contacto, Frontera Arqueológica, Violencia.

\section{Abstract:}

The emergence and spread of Neolithic way of life confronts us with a peculiar world situation. Two radically different forms of life in the economic sphere shared and divided the territory. With only few traces attributable to direct violence between the two factions, we can sense different encounters and conflicts between hunters and farmers in the archaeological record. We try to raise here concepts such as contact resistance or boundary, so help us give a new insight into the group and intragroup relations that occurred at the transition between the Mesolithic and Neolithic. In this time of cohabitation relationships and contacts intend visible, but also forms of conflict often indirect. Anyway, the end of this time of coexistence is clear: the virtual disappearance of life forms purely hunter-gatherer and large-scale adoption, before or after what we understand as forms of Neolithic life.

\section{Key words:}

Neolithic/Mesolithic, Contact, Archaeological Boundaries, Violence.

1 Universidad Complutense de Madrid - carmencorteshistoria@gmail.com 


\section{Introducción}

La dificultad de rastrear el conflicto en la historia puede complicarse cuanto más atrás nos remontemos. Esta aparente pérdida de información no debe asustarnos, sino que debe motivarnos a buscar nuevas propuestas de investigación. En el presente artículo plantearemos la búsqueda de posibles indicios de conflicto dentro de un período histórico muy particular: la transición al Neolítico. Este acontecimiento adquiere especial interés al tener como principales actores dos sociedades distintas en el aspecto social y económico. De una parte, las poblaciones mesolíticas, con una economía de tipo cazador-recolector, de otra, aquellas que comienzan a producir alimentos.

A pesar de tener dos formas de vida diferentes, debemos tener en cuenta que se trata de un momento de transición gradual. Uno de los errores más habituales, en el estudio del período, ha sido el de caracterizarlo como un punto y aparte, como un corte en la historia, en vez de pretender vislumbrar una gradación de todo el proceso que nos arroje algo de luz en toda esta miscelánea.

Los puntos de vista desde los que se ha estudiado el paso al Neolítico han sido diversos, pero tradicionalmente -en el caso europeo- se han centrado en las poblaciones que tecnológicamente eran superiores, señalándolas por ello como más "avanzadas", descuidando el estudio para aquellas que aún mantenían el sistema cazador-recolector. Sin embargo, en las últimas décadas, las nuevas voces teóricas, procedentes de la corriente poscolonialista, parecen haber llegado también al estudio de la transición, objeto de este texto. Varios autores reclaman focalizar la atención en las poblaciones de cazadores que se encuentran en el territorio al que llegan estos nuevos productores y que en muchos textos dejaban de nombrarse (BUDJA, 2009). Nos inclinamos a ello en el presente estudio, a pesar de las acusaciones de algunos autores (PLUCENNICK, 1999: 664) de caer en una visión romántica del proceso al idealizar de alguna manera las formas de vida cazadoras-recolectoras.

El problema continúa al "mitificar" el cambio a la producción de alimentos. Parece que, desde el presente, necesitamos poner un punto de inicio claro, por remoto que sea, a nuestro modo de vida. Podría ser, la sedentarización y la producción de alimentos, diferenciando a éstos del modo de vida cazador-recolector que tanto significado tuvo en tiempos coloniales. Parece que, en el imaginario colectivo occidental, esta transición supone el paso de la civilización a la barbarie (BUDJA, 1999: 121), ese mantra griego que tanto significado ha tenido en diversos momentos de la filosofía occidental.

A tenor de todo esto, y aunque resulte difícil estudiar este campo, nuestras propuestas pretenden ser optimistas y ayudarnos a encontrar, entre toda esta maraña de ideas, diversos indicios que nos ayuden a visualizar improntas más o menos claras de conflicto para este período. Para ello, comenzamos proponiendo el rastreo de los contactos entre las poblaciones mesolíticas y neolíticas. Por 
contacto entendemos cualquier evidencia de interacción entre ambas culturas, siendo en el caso de la arqueología, por lógica de la propia disciplina, de carácter material, como puede ser el intercambio de objetos entre diversas culturas, o la distancia entre sus asentamientos y las características de los mismos. A pesar de esto, planteamos atender activamente a las ausencias materiales, que cobran tanta importancia como las evidencias mismas, porque nos revelan que pudo no haber interacción incluso de una forma consciente. La ausencia, ha sido obviada y denostada en la arqueología repetidamente sin caer en la reflexión que la misma nos aporta.

\section{Teorías de frontera: una revisión de las mismas}

Para entender este tipo de evidencias de contacto, se han formulado las Ilamadas teorías de frontera, especialmente aplicables a aquellas zonas donde se intuyen continuos intercambios, materiales e ideológicos, entre sociedades mesolíticas y neolíticas. La formulación clásica de estas teorías implica la existencia de un largo período de contactos e interacciones entre grupos mesolíticos y neolíticos en una zona concreta (BÓRIC, 2007: 33).

En esta teoría, la distinción entre los grupos que la conforman se centra, de forma casi exclusiva, en sus modos de producción, sin tener en cuenta otros aspectos como puedan ser la etnicidad o la identidad (KOTSAKIS, 2001). Se crea una frontera entre productores de alimentos y cazadores-recolectores, estableciendo diversas redes de intercambio en las que los bienes que circulan no tienen por qué ser exclusivamente materiales. Diversos autores proponen que el intercambio de ideas (FIEDEL y ANTHONY, 2004: 145-146) sería otro punto crucial en estas teorías de frontera, así como el de personas, especialmente de mujeres (ZVELEBIL, 2005: 94; ZVELEBIL, 2006: 186-187; SULGOSTOWSKA, 2006: 200). Varios ejemplos etnológicos actuales apoyan la teoría de que generalmente, el movimiento de mujeres entre ambos grupos, suele ser unidireccional: desde el grupo cazadores-recolectores hacia el de productores. No queremos afirmar que sea el único tipo de intercambio, pero sí el más avalado, como hemos dicho, por contenidos etnográficos. Es el caso por ejemplo de los Hadza, una tribu cazadora-recolectora que habita en ciertas áreas de Tanzania. En su relación de vecindad con un pueblo bantú y por tanto, productor, los Isanzu, nos ilustran la última idea aquí expuesta. Entre las diferentes relaciones que puedan mantener, reseñamos aquí como, en ocasiones, mujeres Hadza se unen en matrimonio a hombres Isanzu y no al revés (MARLOWE, 2012: 255), ratificando así la hipótesis anterior.

Un punto especialmente interesante, a tener en cuenta en el ámbito de las teorías de frontera, es el de la circulación de objetos que, por su escasez en la sociedad receptora, pueden actuar en la misma como bienes de prestigio (ZVELEBIL, 2006: 185), teniendo diversas consecuencias para ella. Ejemplificando esta idea, las cerámicas o los elementos domésticos (a saber, fauna o semillas) en una 
sociedad de cazadores-recolectores podrían considerarse como bienes que sólo pertenecen a algún individuo, en contra de la pretendida propiedad colectiva que tradicionalmente se ha atribuido a estos grupos, empezando a crear los primeros atisbos de propiedad individual dentro de las sociedades.

Si estudiamos a fondo estas teorías, se puede comprobar que, aunque son de fácil adecuación para la explicación del registro, acarrean una gran problemática a la hora de la interpretación de las mismas. De estos enunciados se desprenden una serie de supuestos que pueden hacernos caer en un error de reduccionismo. La dicotomización de los grupos (BÒRIC, 2002), tan separados exclusivamente por su economía puede no hacernos comprender bien la situación (INGOLD, 1985). Comenzábamos este texto hablando de la importancia de ver el proceso de la transición al Neolítico como algo gradual, por lo que muchas veces las economías están en transición, y poner una separación total entre ambas puede hacernos perder objetividad (RAMOS MUÑOZ, 2000: 280). Estamos falseando datos como, por ejemplo, obviar que un porcentaje muy alto de la economía del primer neolítico proviene de recursos salvajes, obtenidos a través de la caza y la recolección.

Se puede extraer también la idea de superioridad de un grupo sobre otro. Parece que los intercambios circulan, como comentábamos, en una única dirección, en la que los productores son los que mayoritariamente aportan en esta transacción. En todo este proceso subyace la idea de 'aculturación' en un sentido positivo, con una noción implícita de progreso (BÓRIC, 2005: 86). No queremos entrar a valorar aquí el término en sí, sino matizar que el único fin del contacto entre ambas sociedades no tiene por qué ser la aculturación de los cazadores ni estos estar absolutamente dispuestos a ello.

Una vez más, el extrapolar ideas de evolución social y expresarlas en términos económicos nos plantea problemas, evocando viejos postulados de la arqueología como el difusionismo o el funcionalismo (FERNÁNDEZ MARTíNEZ, 2006: 87). De nuevo, como en el caso anterior, parece que desgranamos la historia conociendo el final (RAMOS MUÑOZ, 2000: 284), sin atender a que la sedentarización no tiene por qué ser un fin forzado para todas las poblaciones de cazadores, como se expondrá más adelante.

Es así que, en estos contactos que la historiografía nos presenta como igualitarios, subyace -como venimos recalcando- una situación de dominación por parte de un grupo, pero también de superioridad cultural. Es por eso que, a pesar de la importancia y lo interesante de estas teorías, hay que buscar nuevas formulaciones para no caer en reduccionismos culturales. Quizá la separación de estos grupos en categorías que excedan lo puramente económico, especialmente para un tiempo de transición en el que la mezcla de ambos tipos de recursos, salvajes y domésticos, pudiera acercarse más a la realidad, de ahí la importancia que tienen estos contactos para ambas sociedades.

Otro punto de interés para las teorías de frontera, que hemos formulado brevemente, es la existencia de una frontera ideológica. Propuesta por diversos au- 
tores (BUDJA, 1999; HERNANDO, 1999) esta reflexión se torna especialmente interesante ya que quizá sea la respuesta a los problemas inherentes al concepto de frontera que mencionábamos antes. Esta se basa en la necesidad de buscar el principio de lo que se entiende por "civilización" (BUDJA, 1999: 121), que parece haber sido una constante en la obra arqueológica respondiendo científicamente al mito del final del paraíso de la literatura judeocristiana (HERNANDO, 1999: 584). El uso que se ha hecho de la transición al Neolítico de encontrar el punto exacto de paso de lo salvaje a lo civilizado es lo que tratan de apuntar estos estudios sobre frontera ideológica. Es quizá, por esto, que debemos deconstruir teorías que parecen más destinadas a resolver nuestros propios conflictos que los problemas que nos plantea el estudio de las sociedades del pasado.

\section{Conflicto, ¿visible o invisible?}

Una vez planteadas y explicadas, sucintamente, estas teorías, lo que el arqueólogo encuentra son zonas de coexistencia entre ambos modos de vida. En este artículo trataremos de dar algunas pinceladas sobre las posibles situaciones de conflicto que pudieran darse entre ambos. Para ello, reflexionaremos sobre la visibilidad o la invisibilidad de los procesos, ya que, cómo iremos viendo, la ausencia de datos no implica la ausencia de violencia.

Por tanto, para el período que nos interesa, en el que venimos basando este texto, el comienzo del neolítico, lo que entenderíamos por conflicto sería la coexistencia de dos modos de vida y producción diferentes, además de opuestos y enfrentados, que generarían situaciones de enfrentamiento, conllevando en algunos casos la desaparición de los cazadores (JOVER y GARCÍA, 2014: 58). Entre estas situaciones de conflicto, podemos encontrar el enfrentamiento bélico, la dominación por parte de alguno de los grupos, la exclusión y la resistencia.

\section{Conflictos derivados del uso del territorio}

Muchos de los conflictos que se dan en la transición entre el mesolítico y el neolítico pueden derivarse del uso que hacen las poblaciones del territorio (KIM, 2003: 283). Con la llegada de la tecnología neolítica y en muchos casos de nuevas poblaciones que porten ésta, se configurará el espacio de una manera diametralmente diferente a como había estado siendo articulada, lo que facilitará el encuentro entre ambas formas de vida.

Sin entrar en cuestiones de propiedad del territorio, puesto que son conceptos demasiado modernos, lo que sí podemos intuir es que las poblaciones neolíticas irán ocupando las tierras que sean más propicias para la agricultura (BERNABEU AUBÁN: 1996: 51), puesto que, como ya es sabido, no todos los suelos son hábiles para este tipo de actividad. En esta selección de suelos pudieron darse diversos encuentros, ya que quizá estas áreas estuvieran ocupadas tradicionalmente por grupos de cazadores. 
Lo primero que nos viene en mente al pensar en esta idea es la de un enfrentamiento directo entre ambos grupos. Aunque esto podría ser plausible, no encontramos referencias arqueológicas o antropológicas lo suficientemente claras como para ser atribuidas de forma certera a una violencia directa entre poblaciones mesolíticas y neolíticas. A pesar de que encontramos indicios de violencia, ninguno puede ser utilizado con seguridad para argumentar un conflicto directo desde un punto de vista arqueológico o antropológico. Por esto, no negamos que pudieran producirse, pero tampoco podemos afirmarlo desde el registro material, por lo que lo planteamos como una hipótesis a la espera de nuevos datos.

Lo que es probable es que la ocupación de las tierras por los recién llegados, además de algún posible enfrentamiento directo, diera lugar a lo que podemos Ilamar una violencia indirecta. Con la nueva tipología de asentamientos podrían darse dos configuraciones territoriales.

La primera podría dar lugar a un cerco paulatino de los cazadores (JOVER y GARCÍA, 2014: 72) por parte de los productores. De forma intencionada o no, se irían reduciendo los territorios explotados por los primeros, por lo que se verían poco a poco, obligados a una sedentarización forzada, que podría dar lugar a algún tipo de resistencia o contacto cultural, que muy probablemente acabara con la aculturación de estos grupos y la asimilación de las formas de vida neolíticas.

La segunda propuesta implicaría que la llegada de los neolíticos podría dar lugar a una exclusión hacia los cazadores, es decir, a una imposición por parte de los recién llegados para que abandonen sus tierras -exclusión forzada- o una decisión de los propios recolectores a abandonar sus territorios y asentarse en otros -autoexclusión o exclusión decidida-. No es tampoco extraño que decidieran no establecer contactos y utilizar sus formas de vida como forma de resistencia cultural ante el recién Ilegado. Este paulatino proceso de aislamiento, impuesto o no, tendrá consecuencias negativas para estas poblaciones cazadoras y recolectoras, bien porque pierdan el acceso a las mejores tierras y sus recursos, o bien porque finalice con el aislamiento genético y el intercambio de individuos entre los grupos, abocándolos definitivamente a la desaparición (JOVER y GARCíA, 2014: 72).

Como podemos ver, estas teorías podrían responder al clásico interrogante, que nos plantea el registro arqueológico, de a dónde fueron los cazadores, puesto que en algunas zonas desaparecen de forma abrupta. Estos procesos reflejan un claro conflicto que no es directo, como se viene entendiendo generalmente sobretodo en Arqueología. Podemos intuir algunos de estos procesos atendiendo a las distancia entre los asentamientos de ambas culturas, pero también reparando en las ausencias materiales, que nos hablan de la ausencia de contacto, que puede ser consciente y decidida, y con ello un dato imprescindible a tener en cuenta. Estas acciones además pueden clasificarse como violencia, como un conflicto que nace de la presión derivada del uso del territorio entre diferentes grupos.

Un término que está adquiriendo protagonismo en las publicaciones arqueológicas, y que conviene trabajar y reivindicar es el de Resistencia. Este concepto, 
a grandes rasgos, podríamos definirlo como la habilidad para adaptarse a los diferentes presiones para sobrevivir (GONZÁLEZ-RUIBAL, 2014: 22). Se puede apreciar que es un concepto muy interesante para el período objeto de este artículo. Para poder aplicar este término debemos suponer presiones por parte de un grupo hacia otro, es decir, partir de la base de que existe una violencia previa que se manifieste de manera directa o indirecta; arqueológicamente hablando, que este conflicto sea visible o invisible, como ya comentamos antes.

Puede parecer que, al aplicar este concepto, volvemos a caer en las situaciones que antes exponíamos en el caso de las teorías de frontera, pero las diferencias son claras, sobretodo porque asumimos que hay una situación de conflicto previo. No presuponemos la superioridad de ninguno de los grupos, pero, al observar la paulatina expansión de la agricultura y la desaparición de las sociedades colectoras, entendemos que son estas últimas las que debieron "resistir" de una u otra manera ante el avance de estas formas de vida. Esta resistencia sería a su vez, una vía para que estas poblaciones pudieran mantener su identidad tanto material como cultural (FERNÁNDEZ MARTÍNEZ, 2006: 114).

Para ser capaces de distinguir este fenómeno, es necesario distinguir los núcleos de resistencia. Estos lugares, generalmente asentamientos, pueden identificarse por mantener unos patrones culturales propios, diferentes de los que les rodean, a pesar de la presión que estos últimos pueden ejercer en los primeros. Así, en nuestro caso de estudio, deberíamos prestar especial atención a aqueIlos asentamientos de cazadores recolectores con cronologías bastante bajas, que mantienen intactas sus formas de vida a pesar de la paulatina imposición de la agricultura que se va desarrollando a su alrededor. Mantener sus formas de vida se convierte en este caso, en una forma de resistir a las nuevas ideas y modos de producción.

La importancia del rastreo de estos asentamientos, de los lugares a los que hemos denominado núcleos de resistencia, es vital para entender también toda la complejidad del proceso de transición y para comprender las situaciones de conflicto, objeto de nuestro estudio. La forma en la que se establece la resistencia no tiene que ser, una vez más, directa. Es decir, no tienen por qué establecerse enfrentamientos directos para mostrar un fenómeno de resistencia.

El establecimiento de un contacto puede ser maniobra consciente y pensada por parte de las poblaciones cazadoras para perpetuar estos modos de vida. Mediante el contacto con las poblaciones productoras pueden llegar a una especie de acuerdo tácito por medio del cual se respetarían sus modos de vida (RUBIO DE MIGUEL, 1997: 24). ¿A cambio de qué? De establecer un intercambio que beneficiara a ambos grupos. Intercambio que puede basarse en lo que ya se comentaba líneas arriba: cerámicas, domésticos (a saber, plantas cultivadas y animales), elementos de caza, semillas, pieles, personas, etc., pero también lo más importante quizá para apoyar nuestra tesis del contacto como resistencia cultural: la información (GRONENBORG, 1999: 183, RADOVANIC, 2006: 118). La información sobre el territorio podría ser un bien importante que podrían ofrecer los 
cazadores, muy útil para las poblaciones sedentarias-que convertirían a los cazadores en un importante aliado. Nos parecen reflexiones interesantes, que nos pueden ampliar la visión del contacto pacífico, solo ventajoso para los grupos de cazadores y recolectores, en busca de bienes tecnológicamente más avanzados.

\section{Conclusiones}

Para ir recapitulando ideas, debemos partir de que el origen de los conflictos que surgen durante la transición al Neolítico, se deriva del uso del territorio por parte de ambos grupos. Ya hemos explicado que la mayoría de los encuentros se darían en zonas de recursos atractivos, por lo que toda llegada de grupos foráneos provocaría, de manera intencionada o no, una presión sobre los ya establecidos.

Otro de los problemas que hemos ido apuntando de manera sutil es todo lo que subyace alrededor de la idea de domesticación, término íntimamente ligado a un discurso de dominación (HERNANDO, 1994: 138) porque veríamos lo domesticado como culturalmente superior, más evolucionado, términos anticuados ligados a paradigmas de principios del siglo XX. Además, ver dos grupos opuestos, enfrentados desde un punto de vista económico como elemento diferenciador ignorando los problemas culturales e identitarios o de etnicidad nos hace perder objetividad en todo este proceso. En todo este enjambre de ideas trasnochadas, al enfrentar desde un plano económico funcionalista ambos grupos, se sobreentiende continuamente la superioridad de los grupos productores unidos, como decíamos, a la idea de domesticación.

Esto lleva a la disciplina a un cierto Eurocentrismo, ya que el imaginario colectivo se identifica tradicionalmente con el modo de vida sedentario de los productores (culturalmente más avanzados), lo que ha ido asociando la idea de "otro" para los grupos de cazadores y recolectores. Los europeos nos hemos identificado con los productores, recordándonos los cazadores, en muchos casos, a los vecinos de otros continentes (RICHARDS, 2003: 135). Una vez más, aunque estas ideas nos parezcan manidas, muchos de los textos arqueológicos sobre el período siguen evocando en cierta forma estos pensamientos.

Es por esto que venimos repitiendo, que el contacto cultural queda reducido a un argumento tradicionalmente concebido como algo ventajoso porque revela un reduccionismo funcionalista que, como ya hemos explicado, nos explica aparentemente el registro material, pero cuando reflexionamos más sobre los procesos de dichas explicaciones vemos que son vagas e imprecisas como para explicar el comportamiento humano.

A pesar del simplismo, este funcionalismo no es ingenuo. Al fin y al cabo, se sobreentiende que el contacto es beneficioso, pero, ¿para quién? (KIM, 2003: $278,281)$. A pesar de lo que ya se ha expuesto, de esa utilidad inmediata de los productos cerámicos y otros objetos domésticos para los cazadores, al final 
el gran beneficiado de estos contactos sería el de los productores, a priori más poderoso para las teorías de frontera que analizábamos con anterioridad. El beneficio se daría al facilitar, gracias a esta red de intercambios, que los grupos de cazadores y recolectores poco a poco se integraran en los nuevos modos de vida, bien entrando en las nuevas dinámicas de producción, bien desapareciendo progresivamente. Evolución simple y explicación unidireccional de un proceso bastante más complejo, con más actores en juego del que muchos estudios permiten ver.

Son estas reflexiones simplistas las que hacen pensar, como veíamos en el caso de las teorías de frontera, que más que intentar hallar la explicación de lo que pasó, necesitamos buscar una respuesta a ciertos enigmas que, como seres humanos, llevamos algunos miles de años tratando de encontrar un origen. Nos referimos a lo que expusimos en apartados anteriores sobre las fronteras ideológicas. Si queremos ver en este momento una ruptura total, como se desprende de algunos textos, parece que atendemos a nuestras particulares inquietudes sobre nuestro origen, como grupos humanos sedentarios, más que a intentar construir un discurso científico del pasado. Buscar los orígenes de lo que parecen los inicios de la vida sedentaria tiene, con la expulsión del paraíso su respuesta en la mitología judeocristiana (HERNANDO, 1999: 584), pero parece que a nivel científico también tenemos la necesidad de buscarlo.

Por último, para no olvidarnos de la disciplina que nos ocupa: la Arqueología, que prioriza la realidad material, queremos dejar una última reflexión. Venimos también repitiendo a lo largo del texto que, a la hora de buscar contactos en el registro, se debían tener en cuenta las ausencias materiales. Es importante porque, como decíamos, nos indica la no actividad, pero también entraña unos problemas serios de conservación al realizarse mayoritariamente en soportes perecederos, generalmente de composición orgánica. También, al pensar en los problemas de uso y presión del territorio, podemos sacar en claro que habría diversos procesos como los que explicamos de exclusión y autoexclusión que no dejarían huella aparente en el registro material, sino que requiere desarrollar otras vías para su investigación (CRIADO BOADO, 2006).

Esta reflexión ha de inducirnos a apreciar la invisibilidad material de ciertos procesos (JOVER y GARCÍA, 2014: 57), que no implica que no se hayan producido.-Quizá las nuevas investigaciones sobre este período deberían ir encaminadas a visibilizarlos con la ayuda de otras disciplinas -como la antropología- o de herramientas de la Arqueología como son los estudios del territorio o la etnoarqueología. Nuestra misión, como arqueólogos, es intentar reconstruir los hechos del pasado lo más fielmente posible, intentando ser capaces de entender a las sociedades que nos han precedido y alejando la posibilidad de utilizarlas para resolver nuestros propios conflictos, más bien, tratando de visibilizar los suyos. El beneficio es no hacer visiones sesgadas. 


\section{Bibliografía}

BERNABEU AUBÁN, J. (1996): "Indigenismo y migracionismo. Aspectos de la neolitización en la fachada oriental de la Península Ibérica" Trabajos de Prehistoria $33 \mathrm{n}^{\circ} 2,37-54$

BÓRIC, D. (2002): "The Lepenski Vir conundrum: reinterpretation of the Mesolithic and Neolithic sequences in the Danube Gorges" Antiquity 76 (294), 1026-1039

BÓRIC, D. (2005): "Fizzy horizons of change: orientalism and the frontier model of the Meso-Neolithic transition" En Mesolithic Studies at the Beginning of the 21st Century, MILNER, N.P.W (ed.). Oxford: Oxbow, 81-115

BÓRIC, D. (2007): "Mesolithic-Neolithic interactions in the Danube Gorges" Proceedings of the XV World Congress of the International Union for Prehistoric and Protohistoric Sciences, 31-45

BUDJA, M. (1999): "The transition to farming in Mediterranean Europe. An indigenous response" Documenta Praehistorica XXVI, 119-141

BUDJA, M. (2009): "Early Neolithic pottery dispersal and demic diffusion in southeastern Europe" Documenta Praehistorica XXXVI, 117-137

CRIADO BOADO, F. (2006): "¿Se puede evitar la trampa de la subjetividad? Sobre arqueología e interpretación" Complutum vol.17, 247-253

FERNÁNDEZ MARTíNEZ, V. (2006): Una Arqueología Crítica. Crítica Arqueología, Barcelona

FIEDEL, S.J. y ANTHONY, D.W. (2004): “Deerlayers, pathfinders and icemen. Origins of the European Neolithic as seen from the frontier" en Rockman, M. y Steele, J. Colonization of unfamiliar landscape. The archaeology of adaptations. Londres-Nueva York, Routledge, 144-168

GONZÁLEZ-RUIBAL, A. (2014): An Archaeology of Resistance. Materiality and time in an African bordeland Altamira-Press

GRONENBORG, D. (1999): "A variation on a basic theme: the transition to farming in southern central Europe" Journal of World Prehistory 13, n¹2, 123-210

HERNANDO, A. (1994): "El proceso de neolitización, perspectivas teóricas para el studio" Zephyrus XLVI, 123-142

HERNANDO, A. (1999): "El neolítico como clave de la identidad moderna: la difícil interpretación de los cambios y desarrollos culturales" II Congrés del Neolitic a la Península Ibérica SAGVNTVM-PLAV, Extra 2, 583-588

INGOLD, T. (1985): "The Significance of Storage in Hunting Societies" en GAST, M., SIGAUT, F. y BEUTLER,C. (eds.): Les techniques de conservation des graines a long terme. Paris.

JOVER, F. y GARCÍA, G. (2014): "Sobre la neolitización de los grupos mesolíticos en el este de la Península Ibérica: la exclusión como posibilidad" Pyrenae n45 vol.1, 55-88 
KIM, J. (2003): "Land-use conflict and the rate of the transition to agricultural economy: a comparative study of southern Scandinavia and central-western Korea" Journal of archaeological method and theory vol.10 n³, 277-323

KOTSAKIS, K. (2001): "Mesolithic to Neolithic in Greece. Continuity, discontinuity or change of course? Documenta Praehistorica XXVIII , 63-73

MARLOWE, F. (2012): "Why the Hazda are still hunter-gatherer?" En Ethnicity, Huntrer-Gatherers and the "Other" Association or assimilation in Africa, KENT, S. (ed.) Washington DC., Smithsonian Institution Press, 247-275

PLUCIENNICK, M. (1998): "Deconstructing 'the Neolithic' in the Meso-Neolithic Transition" en EDMONDS, M. y RICHARDS, C.(eds.) Understanding the Neolithic of north-western Europe, Cruithne Press, Glasgow 61-83.

RADOVANIC, I. (2006): "Further notes on Mesolithic-Neolithic contacts in the Iron Gates region and the Central Balkans" Documenta Praehistorica XXXIII, 107-124

RAMOS MUÑOZ, J. (2000): "El problema historiográfico de la diferenciación epipaleolítico-neolítico como debate conceptual" Spal vol.9, 279-292

RICHARDS, M. (2003): "The Neolithic invasion of Europe" Annual Review of Anthropology Vol.32, 135-162

RUBIO DE MIGUEL, I. (1997): "El paradigma difusionista y la neolitización de la Península Ibérica: una explicación recurrente" CuPAUAM, 24, 8-58

SULGOSTOWSKA, Z. (2006): "Mesolithic mobility and contacts on areas of the Baltic sea watershed, the Sudety, and Carpatian Mountains" Journal of Anthropological Archaeology 25, 193-203

ZVELEBIL, M. (2005): "Homo habitus: agency, structure and the transformation of tradition in the constitution of the TRB foraging-farming communities in the North European plain" Documenta Praehistorica XXXII, 87-101

ZVELEBIL, M. (2006): "Mobility, contact, and exchange in the Baltic Sea basin 6000-2000 BC" Journal of Anthropological Archaeology 25, 178-192 
